\section{Aortenklappenstenose: TAVI auch bei intermediärem Risiko auf dem Vormarsch}

\author{
Bei Patienten mit symptomatischer Aortenklappenstenose und mittlerem \\ bis hohem Risiko sind die klinischen Ergebnisse nach einer Transkatheter- \\ Aortenklappenimplantation nach zwei Jahren mindestens so gut wie nach \\ operativem Klappenersatz.
}

$\mathrm{D}$ ie Transkatheter-Aortenklappenimplantation (TAVI) ist mittlerweile eine akzeptierte evidenzbasierte Therapie für symptomatische Patienten mit hochgradiger Aortenklappenstenose, die für eine Operation nicht infrage kommen oder die ein hohes perioperatives Risiko aufweisen. Die stete Verbesserung der Technik sowie die steigende Erfahrung von Operateuren hat global zu einer deutlich zunehmenden Anwendung der TAVI bei Patienten mit niedrigerem Operationsrisiko geführt.

In der nun publizierten PARTNER2-Studie wurden 2.032 Patienten mit hochgradiger Aortenklappenstenose und intermediärem Risiko auf die Durchführung einer TAVI oder eines operativen Aortenklappenersatzes randomisiert. Primärer Endpunkt bei einer Nachbeobachtungsdauer von zwei Jahren waren Tod oder schwerwiegender Schlaganfall.

Im Ergebnis zeigte sich die TAVI gegenüber dem operativen Aortenklappenersatz gleichwertig (formal „nicht unterlegen") hinsichtlich der Ereignisraten des primären Endpunkts (19,3\% vs. $21,2 \% ; p=0,25)$. Patienten, bei denen ein transfemoraler TAVI-Zugang gewählt wurde, schnitten im Ergebnis sogar besser ab als die operierten Patienten.
Leon MB et al. Transcatheter or Surgical Aortic Valve Replacement in Intermediate-Risk Patients. N Engl J Med. 2016;374(20):1609-1620

\section{Kommentar}

Die Ergebnisse der PARTNER-2-Studie bestätigen, dass die TAVI das bevorzugte Verfahren zur Behandlung der hochgradigen Aortenklappenstenose bei Patienten mit hohem und auch intermediärem Risiko ist. Dies gilt besonders für Patienten, bei denen ein transfemoraler Zugangsweg für die TAVI möglich ist. Der stete technische Fortschritt sowie die zunehmende Erfahrung implantierender Zentren wird zum Einsatz des Verfahrens in Patientenkollektiven mit weiter sinkendem Risiko führen, ohne dass ein Ende dieser Entwicklung aktuell absehbar ist.

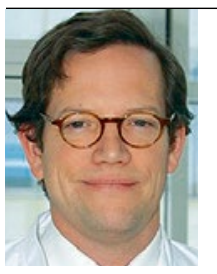

Prof. Dr. med. Peter W. Radke

Klinik für Innere Medizin Kardiologie, Schön Klinik Neustadt

\section{Beeren gegen Erektionsstörungen}

\section{Möglicherweise kann der Verzehr von Obst in Form von Beeren und Zitrusfrüchten zum Erhalt einer guten Erektionsfähigkeit beitragen.}

In die Health Professionals Follow-Up Study wurden 25.096 Männer aus nicht ärztlichen Gesundheitsberufen aufgenommen. Sie waren zu Beginn der Studie um die 60 Jahre alt und hatten zu diesem Zeitpunkt noch keine Erektionsstörungen. Zu Beginn der Studie im Jahr 2000

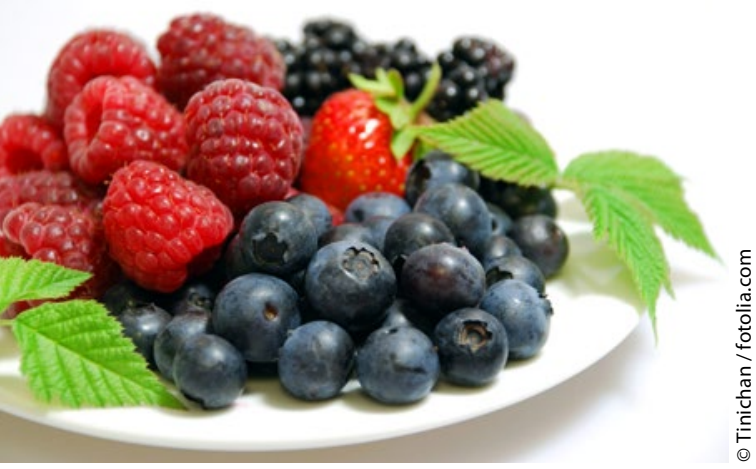

Flavonoide scheinen das Risiko für Erektionsstörungen zu senken. wurde sehr genau dokumentiert, welche dieser Männer eine optimale Erektion für einen zufriedenstellenden Geschlechtsverkehr aufwiesen. Die Befragung wurde 2004 und 2008 wiederholt. Außerdem dokumentierten die Teilnehmer ihre Essgewohnheiten.

In den ersten zehn Studienjahren hatten 35,6\% aller Männer erektile Probleme. In der Gruppe, die hohe Anteile an Anthozyanen, Flavonen und Flavonoiden zu sich nahm, war die Rate der erektilen Dysfunktion deutlich geringer. Ein Flavon-Verzehr im oberen Fünftel war mit einem um $9 \%$ verminderten Risiko für eine erektile Dysfunktion assoziiert (relative Risikoreduktion 0,91). Flavonoide senkten das Risiko um $11 \%$ und Anthocyane um 9\%. Das Fünftel mit dem höchsten Obstverzehr erkrankte um 14\% seltener an einer erektilen Dysfunktion. Die günstigsten Effekte zeigten sich in der
Gruppe, die hohe Anteile an blauroten Beeren zu sich nahm.

Wahrscheinlich ist der hohe Flavonoidanteil von Beeren für diesen Mechanismus verantwortlich. Flavonoide sind sekundäre Pflanzenstoffe, die einen Einfluss auf Farbe und Wirkung eines Nahrungsmittels haben. Sie haben entzündungshemmende, antioxidative sowie antivirale und antibakterielle Wirkungen. Im Tierexperiment schützten Flavonoide vor Dickdarm-, Brust- und Hautkrebs. Chemisch gehören diese Substanzen zur Gruppe der Polyphenole. Flavonoide kommen praktisch in allen Pflanzen vor. Besonders hoch konzentriert sind sie in Beeren. Viele Anthozyane finden sich beispielsweise in Brombeeren, Holunderbeeren, Johannisbeeren und Heidelbeeren und in blauen Trauben.

Bleibt noch $\mathrm{zu}$ erwähnen, dass die Kombination aus einer flavonoidreichen Ernährung mit körperlichem Ausdauertraining überadditiv günstige Effekte zeigte. Der Verzehr von flavonoidhaltigen Früchten in Kombination mit Sport reduzierte das relative Risiko für Erektionsstörungen um $21 \%$. 